\title{
http://bjas.journals.ekb.eg \\ Neonatal Rebound Hyperbilirubinaemia after Intensive Phototherapy in Correlation with Mother Blood Group and Neonatal Weight \\ B.E.M.Hassanein ${ }^{1}$, O.Abu Al-Fotouh ${ }^{1}$, A.M.El Khashab ${ }^{2}$ and M.A.Othman ${ }^{1}$ \\ Pediatrics Dept., Faculty of Medicine, Benha Univ., Benha, Egypt \\ Neonatal Dept., AL Galaa Teaching Hospital , Egypt \\ E-Mail: mohammadbekam@yahoo.com
}

\begin{abstract}
Neonatal jaundice is one of the most common diagnoses in the neonatal period; it is estimated to occur in $60 \%$ of term newborns in the first week of life. In rare instances, the Total Serum Bilirubin (TSB) reaches levels that can cause kernicterus, a condition characterized by bilirubin staining of neurons and neuronal necrosis involving primarily the basal ganglia of the brain and manifested in athetoid cerebral palsy, hearing loss, dental dysplasia, and paralysis of upward gaze, We conducted a prospective study to detect the serum rebound bilirubin among term And preterm newborns diagnosed with neonatal hyperbilirubinaemia who were admitted to the NICU of Al-Galaa Teaching Hospital and NICU of Pediatrics department of Banha University Hospital, requiring double surface, and intensive phototherapy treatment. This was a prospective study to detect the serum rebound bilirubin among100 term \& preterm newborns diagnosed with neonatal hyperbilirubinemia who were admitted to the NICU of Banha University Hospital and AL-Galaa Teaching hospital, requiring double intensive phototherapy treatment. This study is from April 2015 to December 2018, Total Serum Bilirubin $4-48$ hours after discontinuation of phototherapy, significant bilirubin rebound is considered with increased by $2.04 \mathrm{mg} / \mathrm{dL}$ after stopping phototherapy and needing readmission. There were no significant differences between neonates with rebound hyperbilirubinemia and those without as regard birth weight $(\mathrm{P}$ value $=0.745)$, There was no significant difference between discharge level (448hour post intensive photo) (10.0) and after intensive phototherapy level (9.72), we concluded that It is not necessary to keep infants in the hospital to check for rebound because of rare instance of serum billirubin rebound but clinical follow-up 24 to 48 hours later is important furthermore follow up for a risk factor group is a must and keep infant in the hospital should be taken into consideration.
\end{abstract}

Keywords: Neonatal Hyperbilirubinemia, Rebound Bilirubin, Prospective Study, NICU.

\section{Introduction}

Hyperbilirubinaemia is defined as total bilirubin above 95th percentile on the hour specific Bhutani nomogram [1]. Neonatal jaundice is one of the most common diagnoses in the neonatal period; it is estimated to occur in $60 \%$ of term newborns in the first week of life. In rare instances, the Total Serum Bilirubin (TSB) reaches levels that can cause kernicterus, a condition characterized by bilirubin staining of neurons and neuronal necrosis involving primarily the basal ganglia of the brain and manifested in athetoid cerebral palsy, hearing loss, dental dysplasia, and paralysis of upward gaze [2]. Causes of severe neonatal hyperbilirubinaemia are categorized either as hemolytic (blood group mismatch, sepsis, G6PD deficiency) or non-hemolytic (breast feeding jaundice, internal hemorrhage, gestational diabetes, pyloric stenosis, hypothyroidism and some mutations in hepatic enzymes). Prematurity, jaundice in the first 24 hours of life, jaundice noted before discharge from hospital, a history of jaundice treated with phototherapy in siblings and Asian Race are other predisposing factors for severe hyperbilirubinaemia noted by various studies.[3]. For preventing the kernicterus and other complications of hyperbilirubinaemia, jaundice should be managed by phototherapy or exchange transfusion (ECT) [4]. Phototherapy is a useful method because it is easily available and devoid of all complications of double volume ECT, The efficacy of phototherapy depends on the dose and wavelength of light used and the surface area exposed. Despite ECT being an effective method in decreasing TSB level after failing phototherapy, ECT remains or invasive procedure with associated morbidity and mortality. ECT should be considered only when the benefit of decreasing TSB level to prevent kernicterus outweighs the complications associated with the procedure.Intensive phototherapy is a new modality that can rapidly decrease TSB below the threshold for ECT [5]. Underlying alteration in bilirubin production and excretion may persist and cause bilirubin rebound after stopping phototherapy, the need of measurement of bilirubin rebound after stopping phototherapy has been addressed previously by many observational studies[6]. These studies have included neonates born at term or preterm gestation, those with or without positive direct coombs test, and have concluded that significant bilirubin rebound is rare and therefore, measurement of bilirubin rebound is not needed in Addition routine measurement of bilirubin rebound may increase workload add to expenses and prolong the hospital stay[1].

\section{2. patient and methods}

We conducted a prospective study to detect the serum rebound bilirubin among 100 term \& 
preterm newborns diagnosed with neonatal hyperbilirubinemia who were admitted to the NICU of Banhaa Universitiy hospital and ALGalaa Teaching hospital, requiring double intensive phototherapy treatment.This study is from April 2015 to December 2018.

Patients: Newborns male \& female term \& preterm were from the NICU of Al-Galaa Teaching hospital and NICU of Banhaa Universitiy hospital with the following criteria:

Inclusion criteria: Neonatal hyperbilirubinemia.

Exclusion criteria: Neonatal hyperbilirubinemia with cholestasis or obstructive jaundice, and conventional phototherapy

Study tools: All patients were subjected to:

Full clinical assessment through:

1) History

Demographic data: A detailed clinical history including: birth date ,gestational age, sex, maternal drug, feeding type(breast or artificial), age of onset of jaundice, admission and discharge date in addition to any other important risk factor Clinical manifestation :through clinical examination.

\section{2) Laboratory tests including:}

\section{A-Measurement of Serum Total Bilirubin}

The role of the laboratory in the assessment of hyperbilirubinemia is to provide accurate bilirubin results. A number of methods are available on clinical analyzers examination).

\section{B- Laboratory investigations:}

1) Total and direct serum bilirubin.

2) Direct coombs test.

3) Reticulocytic Count.

4) Complete blood count (CBC)

5) Blood group of mother.

6) Blood group of neonate.

7) Total Serum Bilirubin at discontinuation of phototherapy.

8) Total Serum Bilirubin $4-48$ hours after discontinuation of phototherapy, significant bilirubin rebound is considered with increased by $2.3 \mathrm{mg} / \mathrm{dL}$ after stopping phototherapy and needing readmission (Bansal et al., 2010) [5].

9) Serum bilirubin rebound (SBR): $S B R$ is measured 4 to $48 \mathrm{hrs}$ after stopping phototherapy

\section{3) Treatment:}

Including double surface, and intensive phototherapy for 12 to 48 hours treatment to the preterm \& term neonates, and at birth with hyperbilirubinemia respectively. According to the American Academy of Pediatrics. Significant bilirubin rebound was defined as postphototherapy bilirubin level needing re-institution of phototherapy.

\section{Statistical analysis}

The data was tabulated and manipulated according to the Statistical Package for Social Science (SPSS) with the level of significance $\mathrm{p}>0.05$.

\section{Results and discussion}

Mean gestational age of the whole study population was 36 weeks with standard deviation \pm 2 . As regard mean age at presentation, it was 4 days with standard deviation \pm 2 . $60 \%$ of the study population were males while $40.0 \%$ were females table (1). Mean total serum bilirubin showed overall significance between levels on admission, after intensive phototherapy and 48 hours post phototherapy. Post-hoc analysis revealed that mean level on admission was significantly higher (18.29) compared to discharge level (10.0) and after intensive phototherapy level (9.72). There was no significant difference between discharge level (448hour post intensive photo) (10.0) and after intensive phototherapy level (9.72) Table (2). Median reticulocyte count was 3 and ranged from 0.3 to 8.8 . Mean hemoglobin level was 14.5 with standard deviation of \pm 1.9 . Mean hematocrit level was 43.4 with standard deviation of \pm 4.9 Table (3).

There were no significant differences between neonates with rebound hyperbilirubinemia and those without as regard gestational age $(\mathrm{P}$ value $=$ $0.643)$, age at presentation ( $\mathrm{P}$ value $=0.112)$, gender $(\mathrm{P}$ value $=0.669)$ and type of feeding $(\mathrm{P}$ value $=0.471) \quad$ Table (4).

There were no significant differences between neonates with rebound hyperbilirubinemia and those without as regard birth weight $(\mathrm{P}$ value $=$ $0.745)$ Table (5).

Median reticulocyte count was significantly higher in neonates with rebound hyperbilirubinemia (5.7) compared to those without (2.5). P value was 0.001 Table (6)

serum bilirubin (4-48hours) after intensive phototherapy was significantly higher in neonates with rebound hyperbilirubinaemia (12.84) compared to those without $(9.42)$. $\mathrm{P}$ value was 0.002 Table(7).

Total serum bilirubin after intensive phototherapy was significantly higher in neonates with rebound hyperbilirubinemia (12.84) compared to those without (9.42). P value was 0.002 Fig (1). 
Table (1) General characteristics in the whole study group

\begin{tabular}{|c|c|c|}
\hline General characteristics & Mean \pm SD & $36 \pm 2$ \\
\hline Gestational age (weeks) & Mean \pm SD & $4 \pm 2$ \\
\hline Age at presentation (hours) & Males $\quad \mathrm{n}(\%)$ & $60(60.0)$ \\
\hline \multirow[t]{2}{*}{ Gender } & Females $\mathrm{n}(\%)$ & $40(40.0)$ \\
\hline & Mean \pm SD & $2665 \pm 515$ \\
\hline Birth weight (gm) & Yes $\mathrm{n}(\%)$ & $0(0.0)$ \\
\hline Maternal drugs & $\mathrm{AR} \quad \mathrm{n}(\%)$ & $67(67.0)$ \\
\hline Type of feeding & $\mathrm{BF} \quad \mathrm{n}(\%)$ & $33(33.0)$ \\
\hline
\end{tabular}

$$
\mathrm{AR}=\text { Artificial }
$$

$$
\mathrm{BF}=\text { Breast fed }
$$

Table (2) Total serum bilirubin on admission, at discharge and after intensive phototherapy

\begin{tabular}{lccc}
\hline & & Mean \pm SD & P value \\
\hline Total serum bilirubin & On admission $^{\text {a }}$ & $18.29 \pm 4.18$ & $<0.001$ \\
& 4-48hour after intensive $^{\mathrm{b}}$ & $10 \pm 2.9$ & \\
& After intensive $^{\mathrm{b}}$ & $9.72 \pm 2.74$ & \\
\hline
\end{tabular}

Table (3) Reticulocyte count, hemoglobin and hematocrit in the whole study group

\begin{tabular}{lcc}
\hline Blood parameters & & \\
\hline Reticulocyte count & Median (range) & $3(0.3-8.8)$ \\
Hemoglobin & Mean \pm SD & $14.5 \pm 1.9$ \\
Hematocrit & Mean \pm SD & $43.4 \pm 4.9$ \\
\hline
\end{tabular}

Table (4) General characteristics in those with and without rebound hyperbilirubinemia

\begin{tabular}{lcccc}
\hline & & & \multicolumn{2}{c}{ No rebound } \\
& & Rebound(n=91) & (n=91) & P value \\
\hline Gestational age (weeks) & Mean \pm SD & $37 \pm 2$ & $36 \pm 2$ & 0.643 \\
Age at presentation in hours & Mean \pm SD & $3 \pm 2$ & $4 \pm 2$ & 0.112 \\
Gender & Males n $(\%)$ & $6(66.7)$ & $54(59.3)$ & 0.669 \\
& Females n $(\%)$ & $3(33.3)$ & $37(40.7)$ & \\
Type of feeding & AR n (\%) & $7(77.8)$ & $60(65.9)$ & 0.471 \\
& BF n (\%) & $2(22.2)$ & $31(34.1)$ & \\
\hline
\end{tabular}

Table (5) General characteristics in those with and without rebound hyperbilirubinemia

\begin{tabular}{lccc}
\hline & $\begin{array}{c}\text { Rebound } \\
(\mathbf{n}=\mathbf{9})\end{array}$ & $\begin{array}{c}\text { No rebound } \\
(\mathbf{n}=\mathbf{9 1})\end{array}$ & P value \\
\hline Mean \pm SD & $2620 \pm 484$ & $2669 \pm 521$ & 0.745 \\
\hline
\end{tabular}

Table (6) Reticulocyte count, hemoglobin and hematocrit in the whole study group

\begin{tabular}{lcc}
\hline Blood parameters & \\
\hline Reticulocyte count & Median (range) & $3(0.3-8.8)$ \\
Hemoglobin & Mean \pm SD & $14.5 \pm 1.9$ \\
Hematocrit & Mean \pm SD & $43.4 \pm 4.9$ \\
\hline
\end{tabular}

Table (7) Total serum bilirubin in those with and without rebound hyperbilirubinaemia

\begin{tabular}{lcccc}
\hline & & $\begin{array}{c}\text { Rebound } \\
(\mathbf{n = 9 )}\end{array}$ & $\begin{array}{c}\text { No rebound } \\
(\mathbf{n = 9 1 )}\end{array}$ & P value \\
\hline (TSB)on admission & Mean \pm SD & $17.07 \pm 6.08$ & $18.41 \pm 3.97$ & 0.17 \\
(TSB)After intensive & Mean \pm SD & $10.8 \pm 3.7$ & $9.9 \pm 2.8$ & 0.531 \\
(TSB)after intensive & Mean \pm SD & $12.84 \pm 3.35$ & $9.42 \pm 2.48$ & 0.002 \\
(4-48hours) & & & & \\
\hline
\end{tabular}




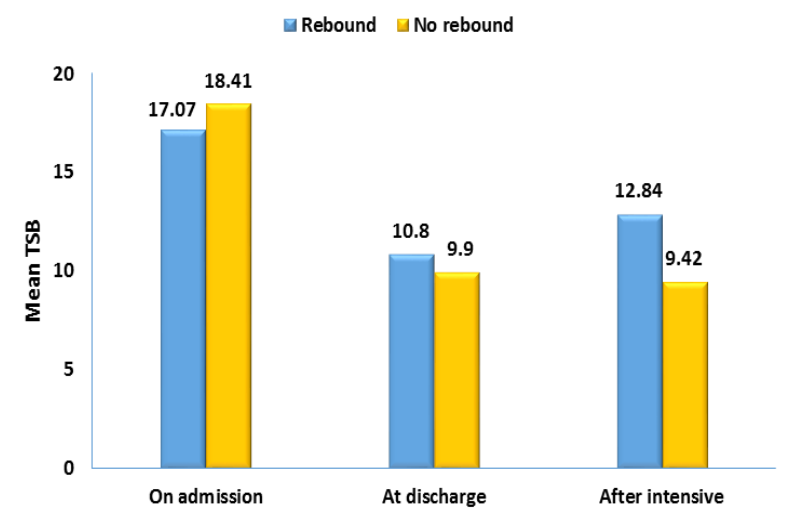

Fig (1) Total serum bilirubin in neonates with rebound hyperbilirubinemia and those without.

Hyperbilirubinemia is the most common condition requiring Evaluation and treatment in newborns. Kernicterus is a preventable brain Injury resulting from severe untreated neonatal hyperbilirubinemia.

Reemergence of kernicterus in a population of term and near-term "healthy" infants after its near eradication is a public health issue [7]. The authors classified significant jaundice according to gestational and postnatal age. Any level exceeding $14 \mathrm{mg} / \mathrm{dl}(240 \mu \mathrm{mol} / \mathrm{l})$ at 4 days in preterm infants and $17 \mathrm{mg} / \mathrm{dl}(290 \mu \mathrm{mol} / \mathrm{l})$ in the term infants was considered serious [8]. Seven incidence studies conducted internationally between 1988 and 2005 identified an estimated incidence of kernicterus at $0.4-2.7$ per 100,000 births.

Studies seem to suggest that some of the ethnic variability in the incidence and severity of neonatal jaundice may have to do with genetic polymorphisms in bilirubin metabolism. Incidence is higher in East Asians and American Indians and lower in African Americans. An interesting finding was reported by Ipek and Bozayakut, showed that Greek neonates born in Greece had more predilections to develop hyperbilirubinemia than those of Greek descent that were born outside of Greece. Evidence is still lacking. In Turkey, a 2004 study showed that significant jaundice occurred in $10.5 \%$ of term infants and in $25.3 \%$ of near-term infants. The authors classified significant jaundice according to gestational and postnatal age.

Neurotoxic levels of bilirubin may vary with postnatal age, maturity of blood-brain barrier, rate of rise of serum bilirubin, serum albumin concentration, presence of hemolysis and comorbidities. The neurotoxic levels of bilirubin in late neonatal period and whether untreated rebound bilirubin may reach those levels are issues for further investigations [5].

The aim of this study is to determine the incidence of post-intensive phototherapy neonatal rebound hyperbilirubinemia need reinstitution of phototherapy and its relation with mother blood group and baby weight Intensive phototherapy Implies the use of high levels of irradiance delivered.

The baby's surface area is exposed as possible. It usually requires at least 2 banks of phototherapy lights or the use of a combination of methods Use special blue fluorescent tubes or specially designed LEDs ifavailable. [1].

Clinical, epidemiologic, and genetic risk factors associated with severe Hyperbilirubinemia include late preterm gestational age, exclusive breastfeeding, glucose-6-phosphate dehydrogenase deficiency, ABO hemolytic disease, East Asian ethnicity, jaundice observed in the first 24 hours of life, cephalohematoma or significant bruising, and history of a previous sibling treated with phototherapy. [1]

In our study, we identified risk factors for significant bilirubin rebound and we found that there was a statistically significant relation between hemolytic disease, low birth weight ,small gestational age, early onset of jaundice, male infant and SBR and that was in concordance with the study done by [5] and [7]. This is due to increased plasma bilirubin in relation to weight and immaturity of liver and liver enzymes responsible for binding and conjugation of bilirubin [9].

Hemolysis was found to be a risk factor for significant bilirubin rebound as 7 out of $9(77.8 \%)$ of our rebound cases were due to hemolytic causes, compared to only 2 cases $(22.2 \%)$ were non hemolytic cases This is due to increased destruction of RBCs and increased bilirubin production [10], which leads to increased bilirubin load in the hepatocytes beyond the capacity of the liver for excretion [11]. However, positive Coomb's test was detected in $11.1 \%$ of rebound cases [5] and [7], found that the presence of Coomb's positive isoimmunisation was significant risk factors for SBR. 
Some studies have studied the incidence of SBR [5], evaluated 232 cases with indirect hyperbilirubinemia and found that the rebound rate was $7.3 \%$.

[1],evaluated 2 groups of infants ,the first group show SBR in $(8.2 \%)$ of cases and the secound group shows SBR in only $(0.7 \%)$ of cases and [5], who evaluated 226 cases found that the rebound rate was $13.3 \%$, while [12] evaluated 375 cases and found that the rate was $5.1 \%$.

cases found that the rate was $4.3 \%$. On the other hand, found no significant differences between mean TSB at the time of termination of phototherapy and $24 \mathrm{hrs}$ and $48 \mathrm{hrs}$ after stopping phototherapy.

We conducted this study to determine the incidence and magnitude of post phototherapy bilirubin rebound needing reinstitution of phototherapy.

Our overviewed study is a prospective one conducted in the neonatal intensive Care unit among neonates among 100 neonate needing phototherapy for significant hyperbilirubinemia during birth hospitalization or re-admission from april 2015 to december 2018

In the present study we have re-measured serum total bilirubin for all the cases of Neonatal hyperbilirubinemia after discontinuation of intensive phototherapy for detection of significant bilirubin rebound 4 to 48 hours post phototherapy for detection of SBR.

the majority of cases developed rebound hyperbilirubinemia as a result of hemolytic disease mostly ABO incompatibility (77.8\%) while $(22.2 \%)$ of the other rebound cases were the result of prematurity and low birth weight which was nearly concordance with shahinaz gamal el din paper, furthermore some cases have more than one risk factor. in addition, it is worth to mention that direct combos test was positive in $(11,1 \%)$ in the rebound cases and positive Reticulocytic count was positive in $(44.4 \%)$ of the rebound cases(median range 5.7)

In the present study risk factors for SBR included gestational age of the mean of $(37 \pm 2)$ weeks The American Academy of Pediatrics considered the gestational age 37-38 weeks is a minor risk factor for development of severe hyperbilirubinemia and the gestational age 35-36 weeks is a major risk factor

Other study revealed that neonates at $<37$ weeks are four times More likely to develop higher serum bilirubin than full term infant.

According to [13], this could be explained by exaggerated Hepatic immaturity and lack of vigorous breast feeding.

onset of jaundice with the mean age of $3 \pm 2$ days which was concordance nearly with [14] reported the age on admission was 5.2 \pm 4.3 days but [15] showed that mean age of onset of jaundice in kernicteric patients was $2.32 \pm 1.3$ days furthermore.

The American Academy of Pediatrics considered the jaundice observed in the first 24 hours as a major risk factor.

our study shows predominance of SBR in males of the percentage of $66.7 \%$ to male and 33.3 to female in addition the male percentage among all the cases of hyperbilirubinemia was $60 \%$ and among the female cases was $40 \%$ which was Concordance with an Egyptian study done in abou al reesh hosptal, the sex distribution among the cases of kernicterus was $61.7 \%$ for males and $38.3 \%$ for females.

( male/female ratio $=1.6: 1$ ) studies done at the NICU of cairo university pediatric hospital as Iskander et al reported $68 \%$ for males and $31.4 \%$ for females, male/female ratio $=(2.16: 1)$

[16] and Hashem et al reported male/female $\operatorname{ratio}(2: 1)$ [15].

In another study done in India there was predominance of males among cases of kernicterus with male/female ratio=2.1:1 (Mala et al., 2015).The same male predominance was reported by Tiker et al in Baskent University, in Turkey [17].

low birth weight was an important risk factor in our study with a mean birth weight of 2669 \pm 521 for the noun rebound cases and $2620 \pm 484$ for the rebound cases which were Concordance with [5]and[7]as all of our study go with it as Well .

furthermore our study mean peak of serum bilirubin was $18.41 \pm 3.97 \mathrm{mg} / \mathrm{dl}$ for noun rebound cases and $17.07 \pm 6.08 \mathrm{mg} / \mathrm{dl}$ for rebound cases which was not Concordance with An Indian study of reported The mean peak of TSB level was $31.73 \pm 10 \mathrm{mg} / \mathrm{dl}$ (ranged from $19-56 \mathrm{mg} / \mathrm{dl}$ ).

In our study artificial feeding was found in (77.8\%)of rebound cases and $(22.2 \%)$ of rebound cases were breast feed.

[5] showed bilirubin increase by $2.3 \mathrm{mg} / \mathrm{dl}$ and 3.8 respectively after stopping phototherapy \& that goes with concordance with our overviewed study as the increase was by $2.04 \mathrm{mg} / \mathrm{dl}$ after stopping phototherapy.

SBR defined as elevated post-phototherapy bilirubin level needing reinstitution of phototherapy [5]

The American pediatrics association now recommends a follow up testing of bilirubin level within 24 hours of discharge in the following neonates, those with hemolytic anemia that received phototherapy, those that received phototherapy early or those neonates who had their phototherapy discontinued when they were 3-4 days old.

based on our data the following predictors should be taken into consideration in selecting the neonate who will need testing for rebound hyperbilirubinemia. Neonates with low birth 
weight, hemolytic etiology, and early onset of jaundice, small gestational age and male infant may be considered if follow up cannot be ensured.

\section{Conclusion}

Extreme hyperbilirubinaemia shows high prevalence in the NICU and still a huge burden in the neonatal unit.

risk factors for Extreme hyperbilirubinaemia and Kernicterus in our population is hemolytic disease, Prematurity, male infant, artificial feeding and low birth weight However a large number of cases still have an unexplained etiology.

Risk factors for significant bilirubin rebound include is hemolytic disease, and some other factor may play rule in predisposing for SBR (prematurity <39 wks., birthweight $<2.600 \mathrm{~kg}$, male infant artificial feeding and onset of jaundice $<72$ hours of age) should be taken into consideration when After the treatment of phototherapy.

hyperbilirubinaemia is still a major problem and can lead to serious problems as kernicterus, previously mentioned risk factor should be taken into consideration in the treatment of hyperbilirubinaemia and SBR should be ruled out when existence of the previously mentioned risk factors.

It is not necessary to keep infants in the hospital to check for rebound because of rare instance of serum bilirubin rebound but clinical follow-up 24 to 48 hours later is important furthermore follow up for a risk factor group is a must and keep infant in the hospital should be taken into consideration.

This prospective study was carried out among 100 neonate to detect the incidence of neonatal SBR after intensive phototherapy and its relation to mother blood group and baby weight in the Neonatal Intensive Care Unit in Benha university hospital and AL-Galaa teaching hospital Who were diagnosed as neonatal hyperbilirubinaemia during the Period from December 2016 to December 2018. Moreover (9\%)of cases in the present study developed SBR after intensive phototherapy and bilirubin increased by the mean of 2.04 after 4 to 48hours of termination of phototherapy. It worth to mention that we used charts which were recommended by the American Academy of Pediatrics but recommenced phototherapy if the rebound bilirubin level was above the value which was appropriate for then patient's age and risk factors.

\section{References}

[1] M.J.Maisels, J.M.Deridder, E.A.Kring, M."Balasubramaniam.Routine transcutaneous bilirubin measurements combined with clinical risk factors improve the prediction of subsequent hyperbilirubinemia". J.Perinatol. Vol 29(9), pp.612-617, Sep 2009.

[2] B.Zabeen, J.Nahar, N.Nabi , A.Baki, S.Tayyeb, K.Azad, et al . Risk factors and outcome of neonatal jaundice in tertiary hospital .J.Ibrahim Med Coll vol.4, pp.7075,2010 .

[3] T.B.Newman,E.Vittinghoff,C.E. McCulloch, T.B Newman, M.J. Maisels. Less aggressive treatment of neonatal jaundice and reports of kernicterus: lessons about practice guidelines. Pediatrics. Vol. 105, pp.242- 245, 2000.

[4] S.H.Behjati, S.Sagheb, S.Aryaseppehr, B.Yaghmai. Adverse events associated with neonatal exchange transfusion for hyperbilirubinaemia.Indian.J.Pediatr. Vol.76, pp.83-85, 2009

[5] A.Bansal, S.Jain, V.RParmar and D.Chawla. Bilirubin rebound after intensive phototherapy for neonatal jaundice.Indian.j.Pediatr. Vol.47, pp.607-609, 2010.

[6] R.J.Yetman, D.KParks, V.Huseby, K.Mistry, I.Garcia. Rebound bilirubin levels in infants receiving phototherapy.J.Pediatr. Vol.133, pp.705-707, 1998.

[7] M.Kaplan, E.Kaplan, C.Hammerman, et al. Post-phototherapy neonatal bilirubin rebound: a potential cause of significant hyperbilirubinaemia. J.Arch Disc Child. Vol 91(1), pp.31-34, Jan 2006.

[8] S.U.Sarici, M.A.Serdar, A.Korkmaz, et al. "Incidence, course, and prediction of hyperbilirubinemia in near-term and term newborns".J.Pediatrics. Vol.113, pp.775-780, 2004.

[9] N.Ambalavanan and W.A.Carlo. Jaundice and Hyperbilirubinemia in the Newborn. In: Kliegman RM, Behrman RE, Jenson HB, Stanton BF, eds. Nelson Textbook of Pediatrics, 19th ed. Philadelphia.Pa. Saunders Elsevier. Vol.96(3), pp. 1943-1962, 2012.

[10] P.Lee, G.Jones, M.J.Seibel. Dua. "Polymorphisms, inUDPglucuronosyltransferas es1A1 and 1A6: a novel mechanism for hyperserotoninaemia in Gilbert's syndrome mimicking carcinoid syndrome. J.Eur Gastroenterol Hepatol. Vol. 19(4), p337-340, Apr 2007.

[11] J.P.Cloherty, M.P.Gregory and Martin CR. Neonatal Hyperbilirubinemia.In. J.P.Cloherty,E.C.Eichenwald,A.R.Hansen, A.R.Stark. Manual of neonatal care, 7th ed. Lippincott Williams \& Wilkins.Vol.26, pp.304-339,2012.

[12] O.Erdeve , U.Tiras , Y.Dallar. Rebound bilirubin measurement is not required for hyperbilirubinaemia regardless of the background attributes of the newborns.J.TropPediatr.Vol. 50,pp 309,2004.

[13] J.M.aisels : Clinical rounds in the Well baby nursery: treating jaundiced babies. Pediatr 
Ann, Vol. 24(10), pp.547-552,1995.

M.I.Maisels, E.Kring. Rebound in serum bilirubin level following intensive phototherapy. Arch Pediatr Adolesc .Med .Vol.156, pp.669-672,2002.

[14] Y.Bao, X.Y.Chen, L.P.Shi, Ma XL, Z.Chen, F.Luo, Z.Y.Zhao. Clinica Features of 116 near Term and Term Infants with AcuteBilirubin Encephalopathy in Eastern China 116.HK.J Paediatr new series. Vol. 18, pp.82-88,2013.

[15] M.Hashem, A.Elwan, A.Ali. M.Noha . Risk factor And Outcome of Kernicterus A threeyears retrospective study in Neonatal intensive care unit in Cairo University.Vol. 65, pp.2389, 2010.

[16] I.Iskander, R.Gamaleldin, M.Kabbani. Root causes for late presentation of severe neonatal hyperbilirubinaemia in Egypt.Vol.18, pp. 1020-3397, 2012.

[17] K.Tiker, H.Kilicdag, A.Tarcan and B.Gurakan. Early Onest Conjugated hyperbilirubinemia in newborn Infants. India. J. indian Pediatr.Vol.73 (5), pp.409-412 (b), 2006. 